\title{
ARTICLE
}

Interventions and public health nutrition

\section{Gestational weight gain targets during the second and third trimesters of pregnancy for women with gestational diabetes mellitus in China}

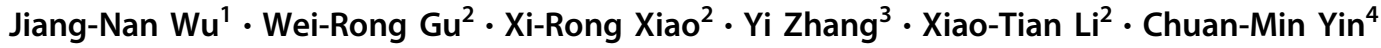

Received: 20 October 2017 / Revised: 29 May 2018 / Accepted: 9 October 2018 / Published online: 30 October 2018

(c) The Author(s) 2018. This article is published with open access

\begin{abstract}
Background/objectives Gestational weight gain (GWG) recommendations for pregnant women with gestational diabetes mellitus (GDM) in China are lacking. The present study aims to examine whether specific GWG targets for women with GDM can improve pregnancy outcomes in comparison with GWG according to the Institute of Medicine (IOM) targets.

Subjects/methods Pregnant women diagnosed with GDM were selected from a retrospective cohort study of 8299 singleton pregnant women aged $18-45$ years in $2012(n=1820)$. GWG ranges were calculated using a receiver operating characteristic (ROC) curve analysis (ROC targets) and the interquartile range (IR) method (the range from the 25th to 75th percentiles of the GWG among GDM women without adverse pregnancy outcomes, IR targets).

Results The incidences of small for gestational age (SGA) births and pregnancy hypertension among women with GDM who gained weight within the ROC targets were lower than the incidences in women who gained weight within the IOM targets (SGA, $7.5 \%$ vs. $8.6 \%$; pregnancy hypertension, $12.6 \%$ vs. $14.1 \%$; both $P<0.05$ ). GWG was associated with a risk of adverse pregnancy outcomes in the total sample (estimated values ranged from -2.95 to 2.08 , all $P<0.05$ ). No statistically significant associations between GWG and adverse pregnancy outcomes were observed in subgroups of pregnant women with appropriate GWGs according to the ROC, IR, and IOM targets. The ROC targets exhibited higher negative predictive values for adverse pregnancy outcomes than the IR and IOM targets.

Conclusion The ROC targets improved pregnancy outcomes and thus represent potential special GWG guidelines for women with GDM in China.
\end{abstract}

These authors contributed equally: Xiao-Tian Li and Chuan-Min Yin

Xiao-Tian Li

xiaotianli555@163.com

$\triangle$ Chuan-Min Yin yincmwujn@126.com

1 Department of Clinical Epidemiology, Obstetrics and Gynecology Hospital of Fudan University, Shanghai 200011, China

2 Department of Obstetrics, Obstetrics and Gynecology Hospital of Fudan University, Shanghai 200011, China

3 Department of Clinical Epidemiology, Children's Hospital of Fudan University, Shanghai 200025, China

4 Department of Nutrition, Obstetrics and Gynecology Hospital of Fudan University, Shanghai 200011, China

\section{Introduction}

Gestational diabetes mellitus (GDM) is defined as "diabetes diagnosed in the second or third trimester of pregnancy that is not clearly overt diabetes," and it is a risk factor for maternal and perinatal complications [1, 2]. The global prevalence of GDM is increasing in parallel with the increase in the prevalence of overweight and obesity among pregnant women, and it is correlated with physical inactivity, dietary patterns, and the sociodemographic characteristics of pregnant women (e.g., advanced maternal age and ethnicity) [3-6].

In China, the prevalence of GDM has rapidly increased since new diagnostic criteria were recommended [7-9]. Based on the new criteria, the prevalence of GDM is $\sim 10 \%$ of pregnancies in municipalities, such as Beijing and Tianjin $[10,11]$. In Shanghai, more than $20 \%$ of pregnant women were diagnosed with GDM in 2012 based on our previous study of 8299 singleton pregnant women [12]. 
Inappropriate gestational weight gains (GWGs), including excessive or insufficient GWGs, are associated with an increased risk of adverse pregnancy outcomes [13-16]. The Institute of Medicine (IOM) published recommendations for GWG in 1990 and revised them in 2009 [17, 18]. The IOM GWG targets are intended for women of all ethnicities and statures and are frequently applied to a specific population, such as women with GDM [18, 19]. However, researchers have not determined whether the IOM targets are applicable to women with GDM who have a greater underlying risk of adverse outcomes.

In a retrospective cohort study of pregnant Australian women with GDM, Wong et al. found that GWG defined according to modified IOM targets did not improve prenatal outcomes among pregnant women with GDM [19]. However, the authors simply subtracted an unwarranted $2 \mathrm{~kg}$ from the upper IOM target or from both the upper and lower targets or copied the range $(0-4 \mathrm{~kg})$ for women with a body mass index $(\mathrm{BMI}) \geq 35 \mathrm{~kg} / \mathrm{m}^{2}$ from another study [19, 20]. These methods for modifying the GWG targets might reduce the reliability of the effect of the targets on adverse pregnancy outcomes. Moreover, the body composition and dietary habits of pregnant Chinese women differ from those of pregnant women from Western countries, indicating that GWG targets differ between Eastern and Western women with GDM [21, 22]. Nevertheless, no studies have been conducted to determine the GWG ranges for Chinese women with GDM.

We conducted a retrospective cohort study of singleton pregnant women with GDM to determine GDM-specific GWG targets for pregnant Chinese women. Because Chinese women are more likely to develop GDM than other populations [23] and women with GDM have a relatively high risk of adverse pregnancy outcomes [19, 24], we hypothesize that GDM-specific GWG targets may improve pregnancy outcomes.

\section{Methods}

\section{Subjects and data collection}

This report is part of a retrospective cohort study of 8299 singleton pregnant women aged 18-45 years who received prenatal examinations and care at the Obstetrics and Gynecology Hospital of Fudan University between January 1 and December 31, 2012. The retrospective cohort study was conducted between 2013 and 2015 to estimate the prevalence of GDM among pregnant women in Shanghai, and was restricted to a review of pregnant women in 2012 because of the time and funding constraints. An oral glucose tolerance test (OGTT) was administered to all pregnant women at 24-28 weeks of gestation to detect
GDM. Pregnant women with a singleton pregnancy who were diagnosed with GDM were included in this study. Exclusion criteria were women with multiple pregnancies and a lack of data on the GDM diagnosis or pregnancy outcomes.

Baseline information, including the maternal age, prepregnancy body weight, height, and parity, was surveyed, and the blood pressure and levels of the fasting blood glucose, triglyceride, cholesterol, high-density lipoprotein, low-density lipoprotein, and DII dimer were measured at the first visit to the hospital. Plasma glucose levels were measured using enzyme-linked colorimetry, triglyceride levels were measured using a free glycerol method, and cholesterol levels were measured using a cholesterol oxidase method. The direct method was used to measure levels of high- and low-density lipoproteins. Levels of the DII dimer were measured using a turbidimetric inhibition immunoassay. Body weight and blood pressure were measured for all subjects at each visit. The mean number of visits was 7.9 (standard deviation, SD, 1.9) and ranged from 2 to 15 visits. Body weight, gestational weeks at labor and birth outcomes (birth weight and Apgar scores at 1 and 5 min after birth) were recorded after delivery. These data were extracted from the electronic medical record systems maintained by the hospital.

\section{Definitions of outcomes and variables}

Weekly GWGs measured during the second and third trimesters were calculated as the GWG from the 12th week of pregnancy until delivery divided by the number of gestational weeks in the second and third trimesters (weeks at labor minus 12 weeks). Pre-pregnancy BMI was calculated as [pre-pregnancy body weight $(\mathrm{kg})] /\left[\right.$ height $\left.^{2}\left(\mathrm{~m}^{2}\right)\right]$. According to the pre-pregnancy BMI classification standard for Chinese adults [25], pregnant women were classified into the following categories: underweight (BMI $<18.5 \mathrm{~kg} /$ $\mathrm{m}^{2}$ ), normal weight (BMI $18.5-23.9 \mathrm{~kg} / \mathrm{m}^{2}$ ), overweight (BMI $24.0-27.9 \mathrm{~kg} / \mathrm{m}^{2}$ ), or obese (BMI $\geq 28 \mathrm{~kg} / \mathrm{m}^{2}$ ). We also used pre-pregnancy BMI classifications based on the IOM standard to classify the women into the following groups: underweight $\left(\mathrm{BMI}<18.5 \mathrm{~kg} / \mathrm{m}^{2}\right)$, normal weight (BMI 18.5-24.9 kg/m²), overweight (BMI $25.0-29.9 \mathrm{~kg} /$ $\mathrm{m}^{2}$ ), and obese $\left(\mathrm{BMI} \geq 30 \mathrm{~kg} / \mathrm{m}^{2}\right)$ [18].

GDM was defined based on a fasting blood glucose level (BGL) $\geq 5.1 \mathrm{mmol} / \mathrm{L}$, a 1 -h $\mathrm{BGL} \geq 10.0 \mathrm{mmol} / \mathrm{L}$, or a 2 -h $\mathrm{BGL} \geq 8.5 \mathrm{mmol} / \mathrm{L}$ after a $75 \mathrm{~g}$ OGTT [26]. The adverse pregnancy outcomes in this study included premature delivery, macrosomia, full-term low birth weight, large for gestational age (LGA), small for gestational age (SGA), neonatal respiratory distress syndrome, and pregnancy hypertension. An infant delivered at $\geq 37$ weeks with a birth weight of $<2500 \mathrm{~g}$ was considered to have a full-term low 


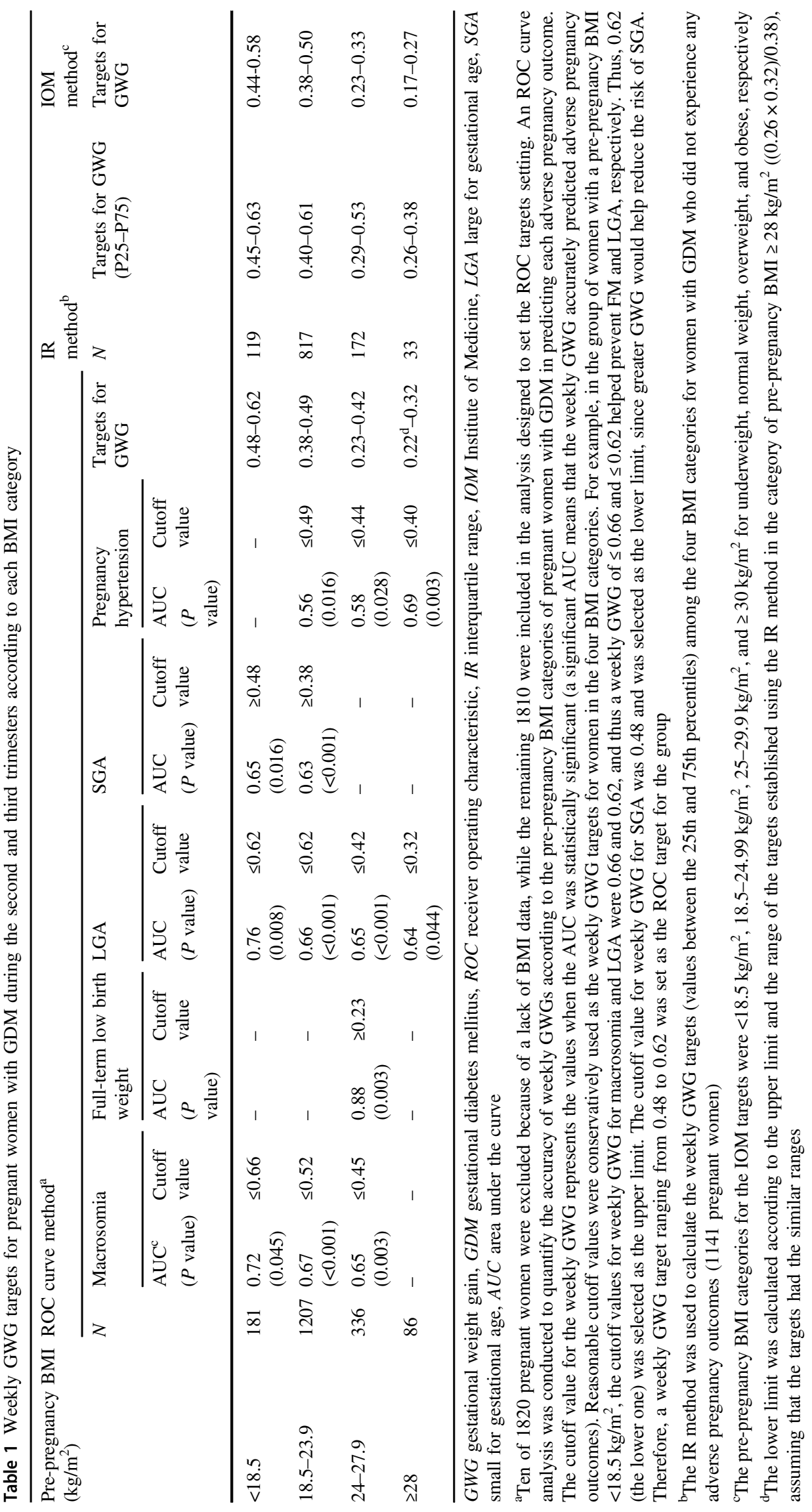


birth weight, and infants with a birth weight of $\geq 4000 \mathrm{~g}$ were considered to have macrosomia [27]. Neonatal respiratory distress syndrome was defined as an infant displaying an Apgar score $\leq 7$ at 1 or 5 min after birth [28]. SGA refers to a standardized birth weight below the 10th percentile (P 10), and LGA refers to a standardized birth weight above P $90[12,19]$. Premature delivery was defined as infants born before 37 weeks of gestation. Pregnant women were diagnosed with pregnancy hypertension when any abnormal blood pressure was detected (systolic blood pressure $\geq 140 \mathrm{mmHg}$ or diastolic blood pressure $\geq 90$ $\mathrm{mmHg}$ ) from the second visit to the end of delivery [12].

\section{Statistical analysis}

The rates of missing variables and outcomes were very low (ranging from 0.05 to $0.5 \%$ ). Therefore, pregnant women for whom data for the variables/outcomes were missing were excluded from the analysis (14 of the 1820 women, $0.8 \%$ ). Continuous variables (weekly GWG, maternal age, pre-pregnancy BMI and gestational weeks) are presented as the means and SD, and categorical variables (parity (1 or $\geq$ 2 ) and the age at pregnancy ( $\geq 35$ years or $<35$ years) are presented as percentages to describe the characteristics of the subjects. Because the weekly GWG is positively correlated with adverse pregnancy outcomes, such as macrosomia, LGA and pregnancy hypertension, and negatively correlated with SGA and full-term low birth weight, receiver operating characteristic (ROC) curve analyses were performed to evaluate the diagnostic value of weekly GWGs measured during the second and third trimesters in predicting the former three adverse pregnancy outcomes and preventing the latter two adverse pregnancy outcomes. A cutoff value for GWG with diagnostic significance for each adverse pregnancy outcome was selected when the integrated area under the ROC curve (AUC) was statistically significant. The lower of the cutoff values for the former three outcomes was chosen as the upper limit, while the higher cutoff value for the latter outcomes was set as the lower limit, constituting the ROC GWG targets (the ROC targets). The interquartile range (IR; range from the 25th to 75th percentiles, P25-P75) of weekly GWGs among pregnant women with GDM for whom adverse pregnancy outcomes were not recorded was calculated across the four BMI categories for Chinese women and set as the IR GWG targets (the IR targets).

Chi-squared analyses (or Fisher's exact test if any cell was $<5)$ were used to assess the statistical significance of difference in the incidences of adverse pregnancy outcomes across the groups of pregnant women with appropriate GWG according to the ROC, IR, and IOM targets (GWG within the targets). Considering the correlation of subjects between the groups, McNemar's paired chi-squared tests were then performed to examine whether the incidences of adverse pregnancy outcomes in women with appropriate GWGs according to the ROC and IR targets differed from women with appropriate GWGs according to the IOM targets. Linear mixed models for repeated measures were used to account for within-subject correlations across the repeatedly measured GWGs and to study the relationship between GWG and adverse pregnancy outcomes. We then repeated linear mixed model analyses in subgroups of pregnant women who exhibited appropriate weight gain according to the ROC, IR, and IOM targets to examine whether the relationship between GWG and adverse pregnancy outcomes changed. Potential factors, such as prepregnancy BMI categories (underweight, normal weight, overweight, or obese), maternal age at pregnancy ( $\geq 35$ years or $<35$ years), parity ( 1 or $\geq 2$ ) and gestational weeks (weeks), were included in the analytical models. Positive and negative predictive values for the cutoff value were calculated for the three targets. A Venn diagram was generated to compare the overlap of pregnant women with appropriate GWGs based on the ROC, IR, and IOM targets.

A two-tailed $P$-value $<0.05$ was considered statistically significant. All data were analyzed using IBM SPSS software, version 19.0 (IBM, Armonk, NY, USA).

\section{Results}

\section{Characteristics of the study population}

In total, 1820 women with GDM were included in the analyses. The average age of the subjects was 29.82 (SD: 3.5) years, the average pre-pregnancy BMI was $22.14 \mathrm{~kg} / \mathrm{m}^{2}$ $\left(4.26 \mathrm{~kg} / \mathrm{m}^{2}\right)$, and the mean gestational age at GDM diagnosis and at labor were 25.9 (14.6) weeks and 39.8 (13.8) weeks, respectively. Among the subjects, $10.10 \%$ were older than 35 years, and $90.69 \%$ were primigravida. The mean weekly GWG during the second and third trimesters of pregnancy was $0.50(0.19) \mathrm{kg}$.

\section{ROC and IR targets}

According to the ROC curve analyses, weekly GWG in the second and third trimesters predicted pregnancy outcomes such as macrosomia, full-term low birth weight, LGA, SGA, and pregnancy hypertension (AUC ranged from 0.63 to 0.88 , all $P<0.05$ ) but not premature delivery or neonatal respiratory distress syndrome (data not shown). The ROC targets were $0.48-0.62 \mathrm{~kg} /$ week for underweight women, $0.38-0.49 \mathrm{~kg} /$ week for normal weight women, $0.23-0.42$ $\mathrm{kg} /$ week for overweight women, and $0.22-0.32 \mathrm{~kg} / \mathrm{week}$ for 
Table 2 Incidences of adverse pregnancy outcomes in women with appropriate gestational weight gain according to the ROC, IR, and IOM targets

\begin{tabular}{|c|c|c|c|c|}
\hline \multirow{3}{*}{$\begin{array}{l}\text { Adverse pregnancy } \\
\text { outcome }\end{array}$} & \multicolumn{4}{|c|}{ Pregnant women with appropriate gestational weight gain } \\
\hline & $\begin{array}{l}\text { ROC targets } \\
(N=477)\end{array}$ & $\begin{array}{l}\text { IR targets } \\
(N=859)\end{array}$ & $\begin{array}{l}\text { IOM targets } \\
(N=560)\end{array}$ & $\begin{array}{l}P \text { value from Chi- } \\
\text { squared tests* }\end{array}$ \\
\hline & $n(\%)$ & $n(\%)$ & $n(\%)$ & \\
\hline Premature delivery & $23(4.8)$ & $39(4.5)^{\dagger}$ & $23(4.1)$ & 0.85 \\
\hline Macrosomia & $17(3.6)$ & $51(5.9)^{\dagger}$ & $22(3.9)$ & 0.08 \\
\hline Full-term low birth weight & $4(0.8)$ & $5(0.6)$ & $4(0.7)$ & 0.86 \\
\hline LGA & $38(8.0)$ & $93(10.8)^{\dagger}$ & $43(7.7)$ & 0.08 \\
\hline SGA & $36(7.5)^{\dagger}$ & $69(8.0)^{\dagger}$ & $48(8.6)$ & 0.83 \\
\hline $\begin{array}{l}\text { Neonatal respiratory } \\
\text { distress syndrome }\end{array}$ & $1(0.2)$ & $1(0.1)$ & $1(0.2)$ & $1.00 * *$ \\
\hline Pregnancy hypertension & $60(12.6)^{\dagger}$ & $125(14.6)^{\dagger}$ & $79(14.1)$ & 0.60 \\
\hline
\end{tabular}

$R O C$ receiver operating characteristic, $I R$ interquartile range, $I O M$ Institute of Medicine, $L G A$ large for gestational age, $S G A$ small for gestational age

${ }^{*} P$ values from chi-squared tests of differences in the incidences of adverse pregnancy outcomes among the three groups

$* * P$ values from Fisher's exact tests

${ }^{\dagger} P<0.05$ from McNemar's chi-squared paired tests compared with the incidence of adverse pregnancy outcomes among pregnant women who exhibited appropriate weight gain according to the IOM targets obese women (Table 1). A total of 1141 pregnant women with GDM who did not experience any adverse pregnancy outcomes were included in the analysis using the IR method. The IR targets were 0.45-0.63, 0.40-0.61, 0.290.53 , and $0.26-0.38 \mathrm{~kg} /$ week for underweight, normal weight, overweight, and obese women, respectively (Table 1).

\section{Comparison of the incidence of adverse pregnancy outcomes}

Statistically significant differences in the incidences of adverse pregnancy outcomes were not observed among the groups of pregnant women with appropriate GWGs according to the ROC, IR, and IOM targets (Table 2). However, McNemar's chi-squared paired tests indicated a significantly lower risk of having SGA infants and pregnancy hypertension for women with appropriate GWGs according to the ROC targets than for women with appropriate GWGs based on the IOM targets (SGA, 7.5\% vs. $8.6 \%$; pregnancy hypertension, $12.6 \%$ vs. $14.1 \%$, both $P<$ $0.05)$. Pregnant women with GDM who had appropriate GWGs according to the IR targets had higher risks of premature delivery, pregnancy hypertension and delivering infants with macrosomia or LGA infants than the women with appropriate GWGs according to the IOM targets. However, the risk of delivering SGA infants was lower in pregnant women who had appropriate GWGs according to the IR targets than in women with appropriate GWGs based on the IOM targets (Table 2).

\section{Relationship between GWG and adverse pregnancy outcomes}

After controlling for potential confounding factors, linear mixed models revealed that the GWG was significantly correlated with premature delivery ( $\beta[95 \%$ confidence interval $]=-1.90 \quad[-2.71$ to -1.09$])$, macrosomia $(2.08$ [1.34 2.81]), full-term low birth weight $(-2.95$ [-4.61 to 1.29]), LGA (2.08 [1.51 to 2.64]), SGA (-1.66 [-2.31 to $1.00])$, and pregnancy hypertension (0.75 [0.24 to 1.26$])$. However, a statistically significant correlation was not observed for neonatal respiratory distress syndrome (Table 3 ). We then repeated the analysis with the models in subgroups of pregnant women who exhibited appropriate weight gains according to the ROC, IR, and IOM targets to examine whether the three targets improved pregnancy outcomes. Statistically significant correlations between the GWG and macrosomia, full-term low birth weight, LGA, SGA, and pregnancy hypertension were not observed, while the GWG was still correlated with premature delivery (Table 3).

\section{Values of the targets in predicting adverse pregnancy outcomes}

The predictive values of the three targets for each adverse pregnancy outcomes were evaluated. Among the three standards, the ROC targets exhibited the best comprehensive predictive value for adverse pregnancy outcomes. The negative predictive values for macrosomia, full-term low birth weight, LGA, SGA, and pregnancy hypertension were 


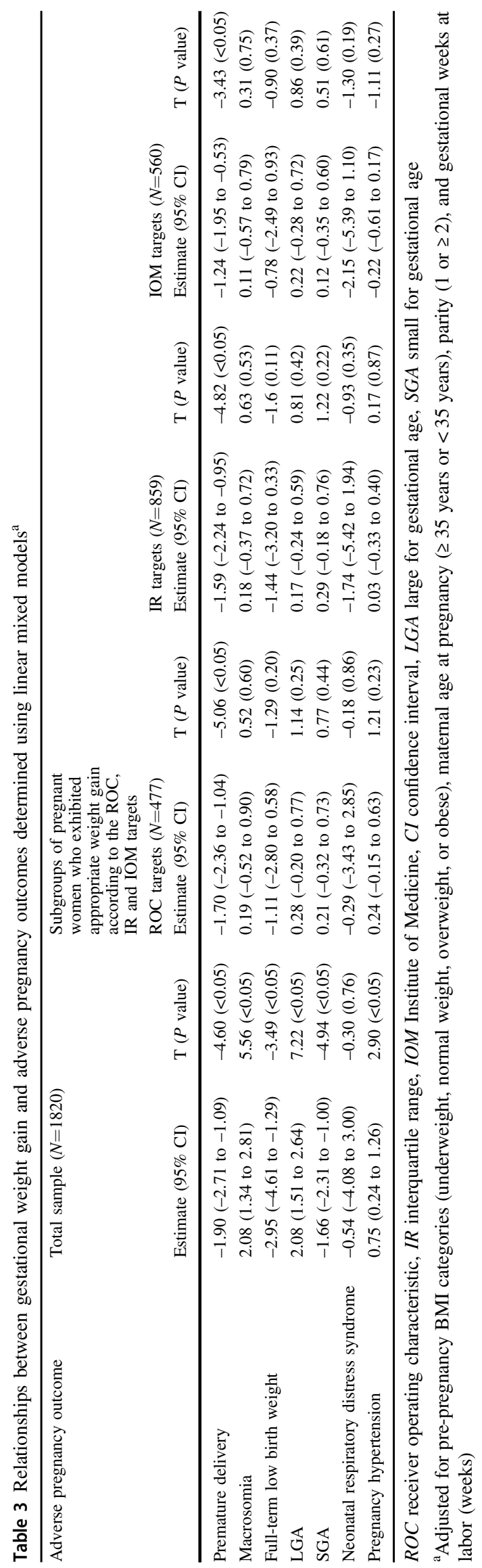

$96.9 \%, 99.3 \%, 93.5 \%, 93.7 \%$, and $87.4 \%$, respectively. The positive predictive values were $10.1 \%, 3.5 \%, 17.3 \%$, $17.5 \%$, and $19.4 \%$, respectively (Table 4 ).

\section{Comparison of the overlap of the three targets}

A total of 1025 pregnant women were identified as having appropriate GWGs according to at least one of the three targets, 32.6\% (334) of which were shared by all three targets (Fig. 1).

\section{Discussion}

In this retrospective cohort study of pregnant women with GDM, GWGs according to the ROC targets during the second and third trimesters improved pregnancy outcomes in women with GDM in Shanghai, China. Women with GDM with exhibiting appropriate GWGs according to the ROC targets had a lower risk of adverse pregnancy outcomes (e.g., SGA and pregnancy hypertension) than women with appropriate GWGs according to the IOM targets, and the negative predictive values of the ROC targets were superior to the IOM targets.

These findings are not consistent with the results of a previous study on Australia women, in which the authors revealed that GWGs defined according to modified stringent targets did not improve pregnancy outcomes [19]. However, the previously reported method for modifying IOM GWG targets has certain deficiencies. According to the Venn diagram, the ROC targets are more stringent than the IOM and IR targets, since the percentage of pregnant women exhibiting appropriate GWG according to the ROC targets outside the intersections of the three targets was markedly lower than the percentage defined by the IOM and IR targets $(1.4 \%, 8.9 \%$, and $37.4 \%$, respectively).

Our findings are similar to the results from previous studies of pregnant Chinese women [21, 22]. The ROC targets for abnormal weight (including pre-pregnancy underweight, overweight, and obese) in women with GDM were slightly higher than the IOM targets. The differences may be attributed to the lower pre-pregnancy BMI criteria for the ROC targets than for the IOM targets and to the observation that Chinese women tend to be thinner than Western women [21, 22, 29]. Both of these aspects may increase the threshold for GWG in Chinese patients with GDM because the pre-pregnancy BMI negatively correlates with GWG [18].

GWGs for overweight and obese women with GDM defined according to the ROC targets were lower than GWGs based on the IOM targets. However, in the present study, $\sim 60 \%$ of the overweight and obese women with GDM experienced excessive weight gain according to the 
Table 4 Predictive values of the ROC, IR and IOM targets for the absence of adverse pregnancy outcomes

\begin{tabular}{|c|c|c|c|c|c|c|c|}
\hline \multirow[t]{2}{*}{ Adverse pregnancy outcome } & \multirow[t]{2}{*}{ Target value ratio } & \multicolumn{3}{|c|}{ Positive predictive value $(\%)$} & \multicolumn{3}{|c|}{ Negative predictive value $(\%)$} \\
\hline & & ROC targets & IR targets & IOM targets & ROC targets & IR targets & IOM targets \\
\hline Macrosomia & $\geq$ upper: < upper & 10.1 & 12.1 & 9.8 & 96.9 & 95.4 & 96.5 \\
\hline Full-term low birth weight & $\leq$ lower: $>$ lower & 3.5 & 3 & 3.7 & 99.3 & 99.3 & 99.2 \\
\hline LGA & $\geq$ upper: < upper & 17.3 & 21.1 & 17.3 & 93.5 & 91.4 & 93.5 \\
\hline SGA & $\leq$ lower: $>$ lower & 17.5 & 15.3 & 17.7 & 93.7 & 93.5 & 93.1 \\
\hline Pregnancy hypertension & $\geq$ upper: < upper & 19.4 & 21.8 & 19.2 & 87.4 & 85.8 & 87.0 \\
\hline
\end{tabular}

$R O C$ receiver operating characteristic, $I R$ interquartile range, $I O M$ Institute of Medicine, $L G A$ large for gestational age, $S G A$ small for gestational age

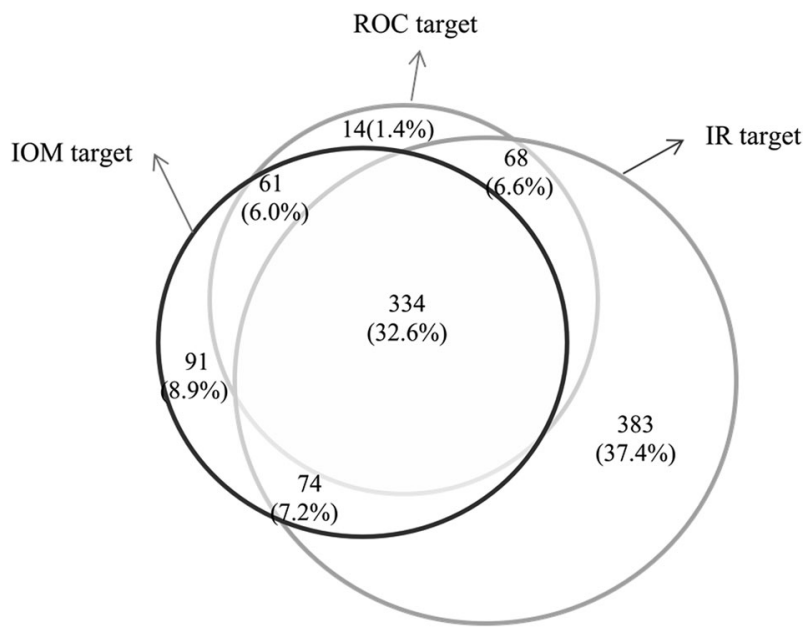

Fig. 1 A Venn diagram showing the comparison of pregnant women with appropriate gestational weight gain based on the ROC, IR, and IOM targets

ROC targets. In addition, the increasing prevalence of overweight and obesity among women of reproductive age and a misunderstanding of GWG due to social influences and views on diet during pregnancy may result in the increasing prevalence of excessive weight gain in pregnant women who are overweight and obese [29-31]. Thus, more attention should be paid to the control of GWG in these groups of women with GDM.

GWG defined according to the IR targets was not associated with adverse pregnancy outcomes, which may be attributed to the deficiency in the method used to calculate IR targets, which was based on a relatively fixed distribution and thus lacked a rational basis for evaluating adverse pregnancy outcomes.

Our study has several limitations. First, the subjects were pregnant women who received care at the Obstetrics and Gynecology Hospital of Fudan University, which may have resulted in an inadequate representativeness of the sample population (subjects may have higher education or income levels, leading to better detection of GDM) and limited the generalizability of the results. However, given the characteristics of GDM and the large sample size (1820 cases), we consider the ROC targets as the meaningful guidelines for GWG in pregnant women with GDM. Second, weight interventions were implemented once the subjects were diagnosed with GDM but not for subjects without GDM. Thus, we were not able to compare our results to findings obtained from women without GDM. Further research is needed to understand the effects of these interventions on both GWG and adverse pregnancy outcomes. Third, the timeline of GWG assessments overlapped with the timeline of a confirmed diagnosis of pregnancy hypertension. Thus, we were not able to justify a causal relationship between these two parameters, which might have affected the conclusion regarding the association between pregnancy hypertension and GWG.

Finally, in the total sample, GWG was associated with adverse pregnancy outcomes, with the exception of neonatal respiratory distress syndrome; however, the association between GWG and pregnancy outcomes (e.g., macrosomia, full-term low birth weight, LGA, SGA, and pregnancy hypertension) was no longer statistically significant in subgroups of women with appropriate GWGs according to the ROC, IR, and IOM targets. According to the results obtained using linear mixed models, women with appropriate GWGs according to the three targets exhibited an effectively reduced risk of adverse outcomes (e.g., macrosomia, full-term low birth weight, LGA, SGA, and pregnancy hypertension), and the data did not support any of the three targets. GWG has a significant impact on adverse pregnancy outcomes, which might be enhanced in the mixed models, resulting in a decreasing effect of different targets on the outcomes. Further studies are needed to distinguish the extent of the impact of GWG itself and the GWG targets on adverse outcomes.

\section{Conclusions}

In summary, compared with the IOM targets, ROC targets provide better GWG guidelines, which can improve 
pregnancy outcomes in women with GDM in Shanghai, China. Studies on the applicability of the ROC targets to pregnant women with GDM in other regions of China should be conducted to determine GDM-specific GWG targets in China.

Acknowledgements We thank the health care providers at the Nutrition Outpatient Clinic of Obstetrics and Gynecology Hospital of Fudan University for their hard work during all processes in this study. We are also grateful to the subjects for their support.

Funding This work was supported by a grant from the National Key Research and Development Program of Reproductive Health \& Major Birth Defects Control and Prevention (No. 2016YFC1000403).

Author contributions $\mathrm{CM}$ Yin and XT Li designed this study and revised the manuscript. JN Wu performed the statistical analyses and wrote the manuscript. Y Zhang, WR Gu, and XR Xiao participated in study development and data collection. All authors have read and approved the final manuscript.

\section{Compliance with ethical standards}

Ethical approval and consent to participate The study was approved by the Ethics Committee of the Obstetrics and Gynecology Hospital of Fudan University (NO. 30-2015). Consent to participate was not obtained because this study employed a retrospective cohort design.

Conflict of interest The authors declare that they have no conflict of interest.

Open Access This article is licensed under a Creative Commons Attribution 4.0 International License, which permits use, sharing, adaptation, distribution and reproduction in any medium or format, as long as you give appropriate credit to the original author(s) and the source, provide a link to the Creative Commons license, and indicate if changes were made. The images or other third party material in this article are included in the article's Creative Commons license, unless indicated otherwise in a credit line to the material. If material is not included in the article's Creative Commons license and your intended use is not permitted by statutory regulation or exceeds the permitted use, you will need to obtain permission directly from the copyright holder. To view a copy of this license, visit http://creativecommons. org/licenses/by/4.0/.

\section{References}

1. Agha-Jaffar R, Oliver N, Johnston D, Robinson S. Gestational diabetes mellitus: dose an effective prevention strategy exist? Nat Rev Endocrinol. 2016;12:533-46.

2. American Diabetes Association. Standard of medical care in diabetes-2016. Diabetes Care. 2016;39:1-119.

3. Aune D, Sen A, Henriksen T, Saugstad OD, Tonstad S. Physical activity and the risk of gestational diabetes mellitus: a systematic review and dose-response meta-analysis of epidemiological studies. Eur J Epidemiol. 2016;31:967-97.

4. Tryggvadottir EA, Medek H, Birgisdottir BE, Geirsson RT, Gunnarsdottir I. Association between healthy maternal dietary pattern and risk for gestational diabetes mellitus. Eur J Clin Nutr. 2016;70:237-42.
5. Anna V, van der Ploeg HP, Cheung NW, Huxley RR, Bauman AE. Sociodemographic correlates of the increasing trend in prevalence of gestational diabetes mellitus in a large population of women between 1995 and 2005. Diabetes Care. 2008;31:228893.

6. Reece EA, Leguizamón G, Wiznitzer A. Gestational diabetes: the need for a common ground. Lancet. 2009;373:1789-97.

7. Du MY, Ma RM, Lao TT, Chen Z, Xiao H, Tian YQ, et al. Early third trimester maternal response to glucose challenge and pregnancy outcome in Chinese women-ralationship between upper distribution level and recommended diagnostic criteria. Eur J Clin Nutr. 2015;68:1133-9.

8. Yin J, Kong AP, Chan JC. Prevention and care programs addressing the growing prevalence of diabetes in China. Curr Diab Rep. 2016;16:130.

9. Zhu WW, Yang HX. Diagnosis of gestational diabetes mellitus in China. Diabetes Care. 2013;36:e76.

10. Shang M, Lin L, Ma L, Yin L. Investigation on the suitability of the International Association of Diabetes and Pregnancy Study Group diagnostic criteria for gestational diabetes mellitus in China. J Obstet Gynaecol. 2014;34:141-5.

11. Leng J, Shao P, Zhang C, Tian H, Zhang F, Zhang S, et al. Prevalence of gestational diabetes mellitus and its risk factors in Chinese pregnant women: a prospective population-based study in Tinajin, China. PLoS ONE. 2015;10:e0121029.

12. Yin CM, Li Q, Zhang Y, Niu DY, Yan WL, Li XT. Association of pre-pregnancy body mass index and gestational weight gain with pregnancy outcomes in Chinese women: a population-based historical cohort study.

13. Goldstein RF, Abell SK, Ranasinha S, Misso M, Boyle JA, Black $\mathrm{MH}$, et al. Association of gestational weight gain with maternal and infant outcomes: a systematic review and mate-analysis. JAMA. 2017;317:2207-25.

14. Xiong C, Zhou A, Cao Z, Zhang Y, Qiu L, Yao C, et al. Association of pre-pregnancy body mass index, gestational weight gain with cesarean section in term deliveries of China. Sci Rep. 2016;6:37168.

15. Muktabhant B, Lawrie TA, Lumbiganon P, Laopaoboon M. Diet or exercise, or both, for preventing excessive weight gain in pregnancy. Cochrane Database Syst Rev. 2015;6:CD007145.

16. Bodnar LM, Siega-Riz AM, Simhan HN, Himes KP, Abrams B. Severe obesity, gestational weight gain, and adverse birth outcomes. Am J Clin Nutr. 2010;91:1642-8.

17. Institute of Medicine Committee on Nutritional Status During Pregnancy and Lactation. Nutrition during pregnancy: Part I weight gain: Part II nutrient supplements. Washington DC: National Academy Press; 1990.

18. Rasmussen KM, Yaktine AL. Weight gain during pregnancy: reexamining the guidelines. Washington DC: National Academy Press; 2009.

19. Wong T, Barnes RA, Ross GP, Cheung NW, Flack JR. Are the Institute of Medicine weight gain targets applicable in women with gestational diabetes mellitus? Diabetologia. 2016; https://doi. org/10.1007/s00125-016-4173-3

20. Kiel DW, Dodson E, Artal R, Boehmer TK, Leet TL. Gestational weight gain and pregnancy outcomes in obese women: how much is enough? Obstet Gynecol. 2007;110:750-8.

21. Wang WP, Chen FF, Mi J, Teng HH. Gestational weight gain and its relationship with the birth weight of offspring. Chin J Obstet Gynecol. 2013;48:321-5.

22. Li YH, Chen XM, Chen SX, Wu JN, Zhou XY, Zheng QL, et al. A cohort study on the impact of pre-pregnancy maternal body mass index, gestational weight gain on neonate birth status and perinatal outcomes in Fujian province. Chin $\mathrm{J}$ Epidemiol. 2014;35:635-9. 
23. Yeung RO, Savu A, Kinniburgh B, Lee L, Dzakpasu S, Nelson C, et al. Prevalence of gestational diabetes among Chinese and South Asians: a Canadian population-based analysis. J Diabetes Complicat. 2017;31:529-36.

24. Blackwell SC, Landon MB, Mele L, Reddy UM, Casey BM, Wapner RJ, et al. Relationship between excessive gestational weight gain and neonatal adiposity in women with mild gestational diabetes mellitus. Obstet Gynecoligy. 2016;128:1325-32.

25. Coorperative Meta-analysis Group of China Obesity Task Force. Predictive values of body mass index and waist circumference to risk factors of related diseases in Chinese adult population. Chin J Epidemiol. 2002;23:5-10.

26. Metzger BE, Gabbe SG, Persson B, Buchanan TA, Catalano PA, Damm $P$, et al. International association of diabetes and pregnancy study groups recommendations on the diagnosis and classification of hyperglycemia in pregnancy. Diabetes Care. 2010;33:676-82.

27. Villar J, Cheikh Ismail L, Victora CG, Ohuma EO, Bertino E, Altman DG, et al. International standards for newborn weight, length, and head circumference by gestational age and sex: the Newborn Cross-Sectional Study of the INTERGROWTH-21st Project. Lancet. 2014;384:857-68.

28. Lagrew DC, Bush MC, McKeown AM, Lagrew NG. Emergent (crash) cesarean delivery: indications and outcomes. Am J Obstet Gynecol. 2006;194:1638-43.

29. Vanstone M, Kandasamy S, Giacomini M, DeJean D, McDonald SD. Pregnant women's perceptions of gestational weight gain: a systematic review and meta-synthesis of qualitative research. Matern Child Nutr. 2016; https://doi.org/10.1111/mcn.12374

30. He JR, Yuan MY, Chen NN, Lu JH, Hu CY, Mai WB, et al. Maternal dietary patterns and gestational diabetes mellitus: a large prospective cohort study in China. Br J Nutr. 2015;113:12921300.

31. Do NC, Secher AL, Cramon P, Ringholm L, Watt T, Damm P, et al. Quality of life, anxiety and depression symptoms in early and late pregnancy in women with pregestational diabetes. Acta Obstet Gynecol Scand. 2016; https://doi.org/10.1111/aogs.13048 